\title{
Descriptor fractional discrete-time linear system with two different fractional orders and its solution
}

\author{
Ł. SAJEWSKI* \\ Faculty of Electrical Engineering, Białystok University of Technology, 45D Wiejska St., 15-351 Bialystok, Poland
}

\begin{abstract}
Factional Discrete-time linear systems with fractional different orders are addressed. The Weierstrass-Kronecker decomposition theorem of the regular pencil is extended to the descriptor fractional discrete-time linear system with different fractional orders. Using the extension, method for finding the solution of the state equation is derived. Effectiveness of the method is demonstrated on a numerical example.
\end{abstract}

Key words: fractional, different order, descriptor, solution.

\section{Introduction}

Descriptor (singular) linear systems have been considered in many papers and books [1-9]. The first definition of the fractional derivative was introduced by Liouville and Riemann at the end of the $19^{\text {th }}$ century $[10,11]$ and another one was proposed in $20^{t h}$ century by Caputo [12]. This idea has been used by engineers for modeling different processes [13-15]. Mathematical fundamentals of fractional calculus are given in the monographs $[10-12,16]$. Solutions of the state equations of descriptor fractional discrete-time linear systems with regular pencils have been given in $[7,17]$ and for continuous-time in $[5,6]$. Reduction and decomposition of singular fractional discrete-time linear systems has been considered in [18]. Application of the Drazin inverse method to analysis of descriptor fractional discrete-time and continuous-time linear systems have been given in $[19,20]$. The positive fractional linear systems has been investigated in $[21,22]$. The positive linear systems with different fractional orders have been addressed in $[23,24]$. Stability of fractional continuous-time linear systems consisting of $n$ subsystem with different fractional orders has been given in [25]. Reachability and minimum energy control problem for systems with two different fractional orders has been considered in [26]. Solution of the state equation of descriptor fractional continuous-time linear systems with two different fractional orders has been introduced in [27]. Comparison of three different methods for finding the solution of the descriptor fractional discrete-time linear system has been given in [28].

In this paper the solution to descriptor fractional discretetime linear systems with two different fractional order is derived.

The paper is organized as follows. In Sec. 2 basic information on the fractional discrete-time linear systems with different fractional orders is recalled. Descriptor fractional discrete-time linear systems with different fractional orders are addressed in Sec. 3, where the Weierstrass-Kronecker de- composition is given. Main idea of the paper is presented in Sec. 4, where the solution to descriptor fractional discretetime linear systems with different fractional orders is derived and illustrated by numerical example. Concluding remarks are given in Sec. 5.

The following notation is used: $\Re$ - the set of real numbers, $\Re^{n \times m}$ - the set of $n \times m$ real matrices, $Z_{+}$- the set of nonnegative integers, $I_{n}-$ the $n \times n$ identity matrix, $A^{T}$ - the transpose matrix $A$.

\section{Fractional different orders discrete-time linear systems}

Consider the fractional discrete-time linear system with two different fractional orders $\alpha$ and $\beta$ of the form

$$
\begin{aligned}
& \Delta^{\alpha} x_{1}(k+1)=A_{11} x_{1}(k)+A_{12} x_{2}(k)+B_{1} u(k), \\
& \Delta^{\beta} x_{2}(k+1)=A_{21} x_{1}(k)+A_{22} x_{2}(k)+B_{2} u(k),
\end{aligned}
$$

where $k \in Z_{+}, x_{1}(k) \in \Re^{n_{1}}$ and $x_{2}(k) \in \Re^{n_{2}}$ are the state vectors, $u(k) \in \Re^{m}$ is the input vector and $A_{i j} \in \Re^{n_{i} \times n_{j}}$, $B_{i} \in \Re^{n_{i} \times m} ; i, j=1,2, n=n_{1}+n_{2}$.

The fractional difference of $\alpha(\beta)$ order is defined by [22]

$$
\begin{gathered}
\Delta^{\alpha} x(k)=\sum_{j=0}^{k}(-1)^{j}\left(\begin{array}{c}
\alpha \\
j
\end{array}\right) x(k-j)=\sum_{j=0}^{k} c_{\alpha}(j) x(k-j), \\
c_{\alpha}(j)=(-1)^{j}\left(\begin{array}{c}
\alpha \\
j
\end{array}\right)=(-1)^{j} \frac{\alpha(\alpha-1) \ldots(\alpha-j+1)}{j !}, \\
c_{\alpha}(0)=1, \quad j=1,2, \ldots
\end{gathered}
$$

\footnotetext{
*e-mail: 1.sajewski@pb.edu.pl
} 
Using (2) we can write Eq. (1) in the matrix form

$$
\begin{gathered}
{\left[\begin{array}{c}
x_{1}(k+1) \\
x_{2}(k+1)
\end{array}\right]=\left[\begin{array}{cc}
A_{1 \alpha} & A_{12} \\
A_{21} & A_{2 \beta}
\end{array}\right]\left[\begin{array}{l}
x_{1}(k) \\
x_{2}(k)
\end{array}\right]} \\
-\sum_{j=2}^{k+1}\left[\begin{array}{cc}
c_{\alpha}(j) I_{n_{1}} & 0 \\
0 & c_{\beta}(j) I_{n_{2}}
\end{array}\right]\left[\begin{array}{l}
x_{1}(k-j+1) \\
x_{2}(k-j+1)
\end{array}\right] \\
+\left[\begin{array}{c}
B_{1} \\
B_{2}
\end{array}\right] u(k),
\end{gathered}
$$

where $A_{1 \alpha}=A_{11}+I_{1} \alpha, A_{2 \beta}=A_{22}+I_{2} \beta$.

Note that, the fractional system (3) is equivalent to the $2 \mathrm{D}$ standard system with increasing number of delays.

Theorem 1. The solution to the fractional system described by Eq. (1) with initial conditions $x_{1}(0)=x_{10}, x_{2}(0)=x_{20}$ is given by

$\left[\begin{array}{l}x_{1}(k) \\ x_{2}(k)\end{array}\right]=\Phi_{k}\left[\begin{array}{l}x_{1}(0) \\ x_{2}(0)\end{array}\right]+\sum_{i=0}^{k-1} \Phi_{k-i-1}\left[\begin{array}{c}B_{1} \\ B_{2}\end{array}\right] u(i)$,

$$
k \in Z_{+},
$$

where $\Phi_{k}$ is defined by

$$
\Phi_{k}=\left\{\begin{array}{r}
I_{n_{1}+n_{2}} \text { for } k=0 \\
A \Phi_{k-1}-D_{1} \Phi_{k-2}-\ldots-D_{k-1} \Phi_{0} \\
\text { for } k=1,2, \ldots, i \\
A \Phi_{k-1}-D_{1} \Phi_{k-2}-\ldots-D_{i} \Phi_{k-i-1} \\
\text { for } k=i+1, i+2, \ldots
\end{array}\right.
$$

and

$$
\begin{gathered}
A=\left[\begin{array}{cc}
A_{1 \alpha} & A_{12} \\
A_{21} & A_{2 \beta}
\end{array}\right], \\
D_{k}=\left[\begin{array}{cc}
c_{\alpha}(k+1) I_{n_{1}} & 0 \\
0 & c_{\beta}(k+1) I_{n_{2}}
\end{array}\right] .
\end{gathered}
$$

Proof is given in [22].

\section{Descriptor fractional different orders discrete-time linear systems}

Consider the descriptor fractional discrete-time linear system with two different fractional orders

$$
\begin{aligned}
& E_{1} \Delta^{\alpha} x_{1}(k+1)=A_{11} x_{1}(k)+A_{12} x_{2}(k)+B_{1} u(k), \\
& E_{2} \Delta^{\beta} x_{2}(k+1)=A_{21} x_{1}(k)+A_{22} x_{2}(k)+B_{2} u(k),
\end{aligned}
$$

where $k \in Z_{+}, x_{1}(k) \in \Re^{n_{1}}$ and $x_{2}(k) \in \Re^{n_{2}}$ are the state vectors, $u(k) \in \Re^{m}$ is the input vector and $E_{i}, A_{i j} \in \Re^{n_{i} \times n_{j}}$, $B_{i} \in \Re^{n_{i} \times m} ; i, j=1,2$.
Similar as in Sec. 2, using (2) we can write Eq. (6) in the matrix form

$$
\begin{gathered}
{\left[\begin{array}{cc}
E_{1} & 0 \\
0 & E_{2}
\end{array}\right]\left[\begin{array}{c}
x_{1}(k+1) \\
x_{2}(k+1)
\end{array}\right]=\left[\begin{array}{cc}
A_{1 \alpha} & A_{12} \\
A_{21} & A_{2 \beta}
\end{array}\right]\left[\begin{array}{l}
x_{1}(k) \\
x_{2}(k)
\end{array}\right]} \\
-\sum_{j=2}^{k+1}\left[\begin{array}{cc}
c_{\alpha}(j) E_{1} & 0 \\
0 & c_{\beta}(j) E_{2}
\end{array}\right]\left[\begin{array}{l}
x_{1}(k-j+1) \\
x_{2}(k-j+1)
\end{array}\right] \\
+\left[\begin{array}{c}
B_{1} \\
B_{2}
\end{array}\right] u(k)
\end{gathered}
$$

where $A_{1 \alpha}=A_{11}+E_{1} \alpha, A_{2 \beta}=A_{22}+E_{2} \beta$ and $E_{1} \in$ $\Re^{n_{1} \times n_{1}}, E_{2} \in \Re^{n_{2} \times n_{2}}$, which represents the descriptor 2D standard system with increasing number of delays.

Finding a solution for the system (7) is very difficult since $\operatorname{det} E_{1}=0, \operatorname{det} E_{2}=0$ and the pencil is very complex.

It is much easier to use a standard form

$$
E\left[\begin{array}{c}
\Delta^{\alpha} x_{1}(k+1) \\
\Delta^{\beta} x_{2}(k+1)
\end{array}\right]=A\left[\begin{array}{l}
x_{1}(k) \\
x_{2}(k)
\end{array}\right]+B u(k),
$$

where

$E=\left[\begin{array}{cc}E_{1} & 0 \\ 0 & E_{2}\end{array}\right], \quad A=\left[\begin{array}{cc}A_{11} & A_{12} \\ A_{21} & A_{22}\end{array}\right], \quad B=\left[\begin{array}{c}B_{1} \\ B_{2}\end{array}\right]$.

It is assumed that

$$
\operatorname{det} E=0
$$

and

$$
\operatorname{det}\left[\left[\begin{array}{cc}
E_{1} z_{1} & 0 \\
0 & E_{2} z_{2}
\end{array}\right]-\left[\begin{array}{cc}
A_{11} & A_{12} \\
A_{21} & A_{22}
\end{array}\right]\right] \neq 0
$$

for some $z \in C$ (the field of complex numbers). This assumption lead to descriptor system with regular pencil.

Now, using the Weierstrass-Kronecker decomposition theorem of the regular pencil $[22,29]$ and adopting it to the systems with two different fractional orders, we have the following Lemma.

Lemma 1. If (9a) and (9b) hold for the systems with two different fractional orders (8), then there exist nonsingular matrices $P, Q \in \Re^{n \times n}$ such that

$$
\begin{gathered}
P\left[\left[\begin{array}{cc}
E_{1} z_{1} & 0 \\
0 & E_{2} z_{2}
\end{array}\right]-\left[\begin{array}{cc}
A_{11} & A_{12} \\
A_{21} & A_{22}
\end{array}\right]\right] Q \\
=\left[\begin{array}{cc}
\bar{E}_{1} z_{1} & 0 \\
0 & \bar{E}_{2} z_{2}
\end{array}\right]-\left[\begin{array}{cc}
\bar{A}_{11} & \bar{A}_{12} \\
\bar{A}_{21} & \bar{A}_{22}
\end{array}\right],
\end{gathered}
$$

where

$$
P=\left[\begin{array}{cc}
P_{1} & 0 \\
0 & P_{2}
\end{array}\right], \quad Q=\left[\begin{array}{cc}
Q_{1} & 0 \\
0 & Q_{2}
\end{array}\right]
$$


Descriptor fractional discrete-time linear system with two different fractional orders and its solution

and the following decomposition is possible

$$
\begin{aligned}
\bar{E}_{1}=P_{1} E_{1} Q_{1} & =\left[\begin{array}{cc}
I_{n_{1}^{1}} & 0 \\
0 & N_{1}
\end{array}\right], \\
\bar{E}_{2}=P_{2} E_{2} Q_{2} & =\left[\begin{array}{cc}
I_{n_{2}^{1}} & 0 \\
0 & N_{2}
\end{array}\right], \\
\bar{A}_{11}=P_{1} A_{11} Q_{1} & =\left[\begin{array}{cc}
\widetilde{A}_{11} & 0 \\
0 & I_{n_{1}^{2}}
\end{array}\right], \\
\bar{A}_{12}=P_{1} A_{12} Q_{2} & =\left[\begin{array}{cc}
\widetilde{A}_{12}^{1} & 0 \\
0 & \widetilde{A}_{12}^{2}
\end{array}\right], \\
\bar{A}_{21}=P_{2} A_{21} Q_{1} & =\left[\begin{array}{cc}
\widetilde{A}_{21}^{1} & 0 \\
0 & \widetilde{A}_{21}^{2}
\end{array}\right], \\
\bar{A}_{22}=P_{2} A_{22} Q_{2} & =\left[\begin{array}{cc}
\widetilde{A}_{22} & 0 \\
0 & I_{n_{2}^{2}}
\end{array}\right],
\end{aligned}
$$

where $N_{1} \in \Re^{n_{1}^{2} \times n_{1}^{2}}, N_{2} \in \Re^{n_{2}^{2} \times n_{2}^{2}}$ are a nilpotent matrices with the index $\mu_{i}, i=1,2$ (i.e. $N_{i}^{\mu_{i}}=0$ and $N_{i}^{\mu_{i}-1} \neq 0$ ), $\widetilde{A}_{11} \in \Re^{n_{1}^{1} \times n_{1}^{1}}, \widetilde{A}_{22} \in \Re^{n_{2}^{1} \times n_{2}^{1}}, \widetilde{A}_{21}^{1} \in \Re^{n_{2}^{1} \times n_{1}^{1}}, \widetilde{A}_{21}^{2} \in$ $\Re^{n_{2}^{2} \times n_{1}^{2}}, \widetilde{A}_{12}^{1} \in \Re^{n_{1}^{1} \times n_{2}^{1}}, A_{12}^{2} \in \Re^{n_{1}^{2} \times n_{2}^{2}}$ and rank $E_{1}=n_{1}^{1}$, $\operatorname{rank} E_{2}=n_{2}^{1}, n_{1}^{1}+n_{1}^{2}=n_{1}, n_{2}^{1}+n_{2}^{2}=n_{2}, n_{1}+n_{2}=n$.

Computation methods for the matrices $P$ and $Q$ have been given e.g. in $[8,9,22]$.

Using Lemma 1 and solution presented in $[6,7]$, the solution $x(k)$ to Eq. (6) with given initial conditions $x(0)$ and an input vector $u(k)$ for $k \in Z_{+}$is derived in the next section.

\section{Solution of the state equation}

Premultiplying the state Eq. (8a) by the matrix $P \in \Re^{n \times n}$ and introducing new state vector

$$
\begin{aligned}
& {\left[\begin{array}{c}
\bar{x}_{1}^{1}(k) \\
\bar{x}_{1}^{2}(k) \\
\bar{x}_{2}^{1}(k) \\
\bar{x}_{2}^{2}(k)
\end{array}\right]=Q^{-1}\left[\begin{array}{c}
x_{1}(k) \\
x_{2}(k)
\end{array}\right],} \\
& \bar{x}_{1}^{1}(k) \in \Re^{n_{1}^{1}}, \quad \bar{x}_{1}^{2}(k) \in \Re^{n_{1}^{2}}, \\
& \bar{x}_{2}^{1}(k) \in \Re^{n_{2}^{1}}, \quad \bar{x}_{2}^{2}(k) \in \Re^{n_{2}^{2}}
\end{aligned}
$$

we obtain

$$
\begin{gathered}
P E Q Q^{-1}\left[\begin{array}{c}
\Delta^{\alpha} x_{1}(k+1) \\
\Delta^{\beta} x_{2}(k+1)
\end{array}\right] \\
=P A Q Q^{-1}\left[\begin{array}{l}
x_{1}(k) \\
x_{2}(k)
\end{array}\right]+P B u(k), \quad k \in Z_{+} .
\end{gathered}
$$

Now, substituting (12) and (13) into (14) we have

$$
\begin{aligned}
& {\left[\begin{array}{cccc}
I_{n_{1}^{1}} & 0 & 0 & 0 \\
0 & N_{1} & 0 & 0 \\
0 & 0 & I_{n_{2}^{1}} & 0 \\
0 & 0 & 0 & N_{2}
\end{array}\right]\left[\begin{array}{c}
\Delta^{\alpha} \bar{x}_{1}^{1}(k+1) \\
\Delta^{\alpha} \bar{x}_{1}^{2}(k+1) \\
\Delta^{\beta} \bar{x}_{2}^{1}(k+1) \\
\Delta^{\beta} \bar{x}_{2}^{2}(k+1)
\end{array}\right] } \\
= & {\left[\begin{array}{cccc}
\widetilde{A}_{11} & 0 & \widetilde{A}_{12}^{1} & 0 \\
0 & I_{n_{1}^{2}} & 0 & \widetilde{A}_{12}^{2} \\
\widetilde{A}_{21}^{1} & 0 & \widetilde{A}_{22} & 0 \\
0 & \widetilde{A}_{21}^{2} & 0 & I_{n_{2}^{2}}
\end{array}\right]\left[\begin{array}{c}
\bar{x}_{1}^{1}(k) \\
\bar{x}_{1}^{2}(k) \\
\bar{x}_{2}^{1}(k) \\
\bar{x}_{2}^{2}(k)
\end{array}\right]+\left[\begin{array}{c}
\widetilde{B}_{1}^{1} \\
\widetilde{B}_{1}^{2} \\
\widetilde{B}_{2}^{1} \\
\widetilde{B}_{2}^{2}
\end{array}\right] u(k) }
\end{aligned}
$$

for $k \in Z_{+}$, where

$$
\begin{array}{ll}
\widetilde{B}_{1}^{1} \in \Re^{n_{1}^{1} \times m}, & \widetilde{B}_{1}^{2} \in \Re^{n_{1}^{2} \times m}, \\
\widetilde{B}_{2}^{1} \in \Re^{n_{2}^{1} \times m}, & \widetilde{B}_{2}^{2} \in \Re^{n_{2}^{2} \times m} .
\end{array}
$$

Lastly, from (8) we can distinguish two subsystems. The standard one

$$
\left[\begin{array}{c}
\Delta^{\alpha} \bar{x}_{1}^{1}(k+1) \\
\Delta^{\beta} \bar{x}_{2}^{1}(k+1)
\end{array}\right]
$$$$
=\left[\begin{array}{cc}
\widetilde{A}_{11} & \widetilde{A}_{12}^{1} \\
\widetilde{A}_{21}^{1} & \widetilde{A}_{22}
\end{array}\right]\left[\begin{array}{l}
x_{1}^{1}(k) \\
x_{2}^{1}(k)
\end{array}\right]+\left[\begin{array}{c}
\widetilde{B}_{1}^{1} \\
\widetilde{B}_{2}^{1}
\end{array}\right] u(k)
$$

and nilpotent one

$$
\begin{gathered}
{\left[\begin{array}{cc}
N_{1} & 0 \\
0 & N_{2}
\end{array}\right]\left[\begin{array}{c}
\Delta^{\alpha} \bar{x}_{1}^{2}(k+1) \\
\Delta^{\beta} \bar{x}_{2}^{2}(k+1)
\end{array}\right]} \\
=\left[\begin{array}{cc}
I_{n_{1}^{2}} & \widetilde{A}_{12}^{2} \\
\widetilde{A}_{21}^{2} & I_{n_{2}^{2}}
\end{array}\right]\left[\begin{array}{c}
x_{1}^{2}(k) \\
x_{2}^{2}(k)
\end{array}\right]+\left[\begin{array}{c}
\widetilde{B}_{1}^{2} \\
\widetilde{B}_{2}^{2}
\end{array}\right] u(k) .
\end{gathered}
$$

Using Theorem 1, a solution to the subsystem (17) can be computed by the use of the following formula

$$
\left[\begin{array}{c}
\bar{x}_{1}^{1}(k) \\
\bar{x}_{2}^{1}(k)
\end{array}\right]=\Phi_{k}\left[\begin{array}{c}
\bar{x}_{1}^{1}(0) \\
\bar{x}_{2}^{1}(0)
\end{array}\right]+\sum_{i=0}^{k-1} \Phi_{k-i-1}\left[\begin{array}{c}
\widetilde{B}_{1}^{1} \\
\widetilde{B}_{2}^{1}
\end{array}\right] u(i),
$$

$$
k \in Z_{+},
$$

where

$$
\begin{aligned}
& \Phi_{k}=\left\{\begin{array}{r}
I_{n_{1}^{1}+n_{2}^{1}} \text { for } k=0, \\
\widetilde{A} \Phi_{k-1}-D_{1} \Phi_{k-2}-\ldots-D_{k-1} \Phi_{0} \\
\text { for } k=1,2, \ldots, i, \\
\widetilde{A} \Phi_{k-1}-D_{1} \Phi_{k-2}-\ldots-D_{i} \Phi_{k-i-1} \\
\text { for } k=i+1, i+2, \ldots,
\end{array}\right. \\
& \widetilde{A}=\left[\begin{array}{cc}
\widetilde{A}_{1 \alpha} & \widetilde{A}_{12}^{1} \\
\widetilde{A}_{21}^{1} & \widetilde{A}_{2 \beta}
\end{array}\right] !, \\
& D_{k}=\left[\begin{array}{cc}
c_{\alpha}(k+1) I_{n_{1}^{1}} & 0 \\
0 & c_{\beta}(k+1) I_{n_{2}^{2}}
\end{array}\right] \text {, } \\
& \widetilde{A}_{1 \alpha}=\widetilde{A}_{11}+I_{n_{1}^{1}} \alpha, \quad \widetilde{A}_{2 \beta}=\widetilde{A}_{22}+I_{n_{2}^{1}} \beta .
\end{aligned}
$$


To find a solution of the subsystem (18) for $N_{1} \neq 0, N_{2} \neq 0$ nilpotent (e.g. for

$$
N=\left[\begin{array}{lll}
0 & 1 & 0 \\
0 & 0 & 1 \\
0 & 0 & 0
\end{array}\right]
$$

we have three equations with three unknown elements) we simple start by solving the equation related with the zero row and then continue solving the rest of the equations, see e.g. $[6,7]$.

If $N_{1}=0, N_{2}=0$ then from (18) we have

$$
\begin{gathered}
{\left[\begin{array}{c}
\bar{x}_{1}^{2}(k) \\
\bar{x}_{2}^{2}(k)
\end{array}\right]=\left[\begin{array}{c}
{\left[I_{n_{1}^{2}}-\widetilde{A}_{12}^{2} \widetilde{A}_{21}^{2}\right]^{-1}\left[\widetilde{A}_{12}^{2} \widetilde{B}_{2}^{2}-\widetilde{B}_{1}^{2}\right]} \\
{\left[I_{n_{2}^{2}}-\widetilde{A}_{21}^{2} \widetilde{A}_{12}^{2}\right]^{-1}\left[\widetilde{A}_{21}^{2} \widetilde{B}_{1}^{2}-\widetilde{B}_{2}^{2}\right]}
\end{array}\right] u(k),} \\
k \in Z_{+} .
\end{gathered}
$$

Finally, knowing

$$
\left[\begin{array}{l}
\bar{x}_{1}^{1}(k) \\
\bar{x}_{2}^{1}(k)
\end{array}\right] \quad \text { and } \quad\left[\begin{array}{l}
\bar{x}_{1}^{2}(k) \\
\bar{x}_{2}^{2}(k)
\end{array}\right],
$$

from (13), we can find the desired solution of the system (6) in the form

$$
\left[\begin{array}{l}
x_{1}(k) \\
x_{2}(k)
\end{array}\right]=Q\left[\begin{array}{c}
\bar{x}_{1}^{1}(k) \\
\bar{x}_{1}^{2}(k) \\
\bar{x}_{2}^{1}(k) \\
\bar{x}_{2}^{2}(k)
\end{array}\right], \quad k \in Z_{+}
$$

Remark 1. In this study, only the diagonal form of matrix $E$ has been considered. These considerations can be extended to the systems with the matrix $E$ of (8) in general form. It is well-known [29, 30] that by the use of elementary row operations it is always possible to reduce matrix to its diagonal form.

Examples 1. Find the solution of the descriptor fractional linear system (6) with the fractional orders $\alpha=0.5, \beta=0.6$, matrices

$$
\begin{aligned}
E_{1}= & {\left[\begin{array}{lll}
1 & 0 & 0 \\
0 & 1 & 0 \\
0 & 0 & 0
\end{array}\right], \quad E_{2}=\left[\begin{array}{ccc}
-1 & -1 & -1 \\
2 & 4 & 2 \\
1 & 4 & 1
\end{array}\right], } \\
A_{11}= & {\left[\begin{array}{lll}
1 & 0 & 1 \\
0 & 1 & 0 \\
-1 & 0 & -1
\end{array}\right], A_{12}=\left[\begin{array}{ccc}
4 & 11 & 6 \\
2 & 5 & 2 \\
0 & 0 & -2
\end{array}\right], } \\
A_{21}= & {\left[\begin{array}{lll}
3 & 2 & 6 \\
9 & 2 & 3 \\
3 & 7 & 0
\end{array}\right], A_{22}=\left[\begin{array}{ccc}
0.8 & 1.7 & 2.8 \\
0.4 & 0.8 & 1.4 \\
2.2 & 4.6 & 2.2
\end{array}\right], } \\
B_{1}= & {\left[\begin{array}{c}
1 \\
0 \\
-1
\end{array}\right], B_{2}=\left[\begin{array}{l}
1 \\
0 \\
1
\end{array}\right], }
\end{aligned}
$$

and consistent initial conditions $x_{1}(0)=\left[\begin{array}{lll}1 & 2 & -1\end{array}\right]^{T}$, $x_{2}(0)=\left[\begin{array}{lll}0 & 2 & 1\end{array}\right]^{T}$.
It is easy to check that the matrices (23) satisfy the assumptions (9). In this case the matrices $P$ and $Q$ have the form

$$
P=\left[\begin{array}{cc}
P_{1} & 0 \\
0 & P_{2}
\end{array}\right], \quad Q=\left[\begin{array}{cc}
Q_{1} & 0 \\
0 & Q_{2}
\end{array}\right],
$$

$P_{1}=\left[\begin{array}{ccc}0 & 1 & 0 \\ 1 & 0 & 1 \\ 0 & 0 & -1\end{array}\right], \quad P_{2}=\frac{1}{11}\left[\begin{array}{ccc}1 & -2 & 5 \\ -2 & 4 & 1 \\ 4 & 3 & -2\end{array}\right]$,

$$
Q_{1}=\left[\begin{array}{ccc}
0 & 1 & 0 \\
1 & 0 & 0 \\
0 & -1 & 1
\end{array}\right], \quad Q_{2}=\left[\begin{array}{ccc}
-2 & 1 & -1 \\
1 & 0 & 0 \\
0 & 0 & 1
\end{array}\right]
$$

and

$P E Q=\left[\begin{array}{cccc}I_{n_{1}^{1}} & 0 & 0 & 0 \\ 0 & N_{1} & 0 & 0 \\ 0 & 0 & I_{n_{2}^{1}} & 0 \\ 0 & 0 & 0 & N_{2}\end{array}\right]=\left[\begin{array}{cccccc}1 & 0 & 0 & 0 & 0 & 0 \\ 0 & 1 & 0 & 0 & 0 & 0 \\ 0 & 0 & 0 & 0 & 0 & 0 \\ 0 & 0 & 0 & 1 & 0 & 0 \\ 0 & 0 & 0 & 0 & 1 & 0 \\ 0 & 0 & 0 & 0 & 0 & 0\end{array}\right]$,

$$
Q^{-1}\left[\begin{array}{c}
x_{1}(k) \\
x_{2}(k)
\end{array}\right]=\left[\begin{array}{c}
\bar{x}_{1}^{1}(k) \\
\bar{x}_{1}^{2}(k) \\
\bar{x}_{2}^{1}(k) \\
\bar{x}_{2}^{2}(k)
\end{array}\right]=\left[\begin{array}{c}
\bar{x}_{11}^{1}(k) \\
\bar{x}_{12}^{1}(k) \\
\bar{x}_{11}^{2}(k) \\
\bar{x}_{21}^{1}(k) \\
\bar{x}_{22}^{1}(k) \\
\bar{x}_{21}^{2}(k)
\end{array}\right],
$$$$
P A Q=\left[\begin{array}{cccc}
\widetilde{A}_{11} & 0 & \widetilde{A}_{12}^{1} & 0 \\
0 & I_{n_{1}^{2}} & 0 & \widetilde{A}_{12}^{2} \\
\widetilde{A}_{21}^{1} & 0 & \widetilde{A}_{22} & 0 \\
0 & \widetilde{A}_{21}^{2} & 0 & I_{n_{2}^{2}}
\end{array}\right]
$$

$$
=\left[\begin{array}{cccccc}
1 & 0 & 0 & 1 & 2 & 0 \\
0 & 0 & 0 & 3 & 4 & 0 \\
0 & 0 & 1 & 0 & 0 & 2 \\
3 & 0 & 0 & 0.1 & 1 & 0 \\
1 & 3 & 0 & 0 & 0.2 & 0 \\
0 & 0 & 3 & 0 & 0 & 1
\end{array}\right]
$$

$$
P B=\left[\begin{array}{c}
\widetilde{B}_{1}^{1} \\
\widetilde{B}_{2}^{1} \\
\widetilde{B}_{1}^{2} \\
\widetilde{B}_{2}^{2}
\end{array}\right]=\left[\begin{array}{c}
0 \\
0 \\
1 \\
0.545 \\
-0.091 \\
0.182
\end{array}\right]
$$

$$
n_{1}^{1}=n_{2}^{1}=2, \quad n_{1}^{2}=n_{2}^{2}=1
$$

$$
n_{1}=n_{2}=3, \quad n=n_{1}+n_{2}=6 \text {. }
$$


Taking under considerations $n_{1}^{1}, n_{2}^{1}$, formula (19) has the form

$$
\begin{gathered}
{\left[\begin{array}{l}
\bar{x}_{11}^{1}(k) \\
\bar{x}_{12}^{1}(k) \\
\bar{x}_{21}^{1}(k) \\
\bar{x}_{22}^{1}(k)
\end{array}\right]=\Phi_{k}\left[\begin{array}{c}
\bar{x}_{11}^{1}(0) \\
\bar{x}_{12}^{1}(0) \\
\bar{x}_{21}^{1}(0) \\
\bar{x}_{22}^{1}(0)
\end{array}\right]+\sum_{i=0}^{k-1} \Phi_{k-i-1}\left[\begin{array}{c}
\widetilde{B}_{11}^{1} \\
\widetilde{B}_{12}^{1} \\
\widetilde{B}_{21}^{1} \\
\widetilde{B}_{22}^{1}
\end{array}\right] u(i),} \\
k \in Z_{+},
\end{gathered}
$$

where $\Phi_{k}$ is defined by (20a) with

$$
\begin{aligned}
& \widetilde{A}=\left[\begin{array}{cc}
\widetilde{A}_{1 \alpha} & \widetilde{A}_{12}^{1} \\
\widetilde{A}_{21}^{1} & \widetilde{A}_{2 \beta}
\end{array}\right]=\left[\begin{array}{cccc}
1.5 & 0 & 1 & 2 \\
0 & 0.5 & 3 & 4 \\
3 & 0 & 0.7 & 1 \\
1 & 3 & 0 & 0.8
\end{array}\right] \\
& D_{i}=\left[\begin{array}{cc}
c_{\alpha}(i+1) I_{2} & 0 \\
0 & c_{\beta}(i+1) I_{2}
\end{array}\right] \text {, } \\
& {\left[\begin{array}{l}
\widetilde{B}_{11}^{1} \\
\widetilde{B}_{12}^{1} \\
\widetilde{B}_{21}^{1} \\
\widetilde{B}_{22}^{1}
\end{array}\right]=\left[\begin{array}{c}
0 \\
0 \\
0.545 \\
-0.051
\end{array}\right]}
\end{aligned}
$$

To compute (26), first we have to compute matrices $\Phi_{k}$ for $k \in Z_{+}$, which in this example have the form

$$
\begin{gathered}
\Phi_{0}=I_{4}, \\
\Phi_{1}=\left[\begin{array}{cc}
\widetilde{A}_{1 \alpha} & \widetilde{A}_{12}^{1} \\
\widetilde{A}_{21}^{1} & \widetilde{A}_{2 \beta}
\end{array}\right]=\left[\begin{array}{cccc}
1.5 & 0 & 1 & 2 \\
0 & 0.5 & 3 & 4 \\
3 & 0 & 0.7 & 1 \\
1 & 3 & 0 & 0.8
\end{array}\right], \\
\Phi_{2}=\left[\begin{array}{cccc}
\widetilde{A}_{1 \alpha} & \widetilde{A}_{12}^{1} \\
\widetilde{A}_{21}^{1} & \widetilde{A}_{2 \beta}
\end{array}\right]^{2}-\left[\begin{array}{ccc}
\frac{\alpha(\alpha-1)}{2 !} & I_{2} & 0 \\
0 & \frac{\beta(\beta-1)}{2} I_{2}
\end{array}\right] \\
=\left[\begin{array}{cccc}
7.375 & 6 & 2.2 & 5.6 \\
13 & 12.375 & 3.6 & 8.2 \\
7.6 & 3 & 3.64 & 7.5 \\
2.3 & 3.9 & 10 & 14.79
\end{array}\right], \\
\Phi_{3}=\widetilde{A}_{2}-D_{1} \widetilde{A}-D_{2} I_{4} \\
=\left[\begin{array}{cccc}
23.263 & 19.8 & 27.059 & 45.718 \\
38.5 & 30.663 & 53.077 & 86.236 \\
30.177 & 24 & 19.043 & 36.978 \\
48.359 & 46.677 & 21 & 41.941
\end{array}\right], \ldots
\end{gathered}
$$

then we can commute vector

$$
\left[\begin{array}{llll}
\bar{x}_{11}^{1}(k) & \bar{x}_{12}^{1}(k) & \bar{x}_{21}^{1}(k) & \bar{x}_{22}^{1}(k)
\end{array}\right]^{T} .
$$

Nilpotent subsystem (18) has the form

$$
\begin{gathered}
{\left[\begin{array}{cc}
N_{1} & 0 \\
0 & N_{2}
\end{array}\right]=\left[\begin{array}{cc}
I_{n_{1}^{2}} & \widetilde{A}_{12}^{2} \\
\widetilde{A}_{21}^{2} & I_{n_{2}^{2}}
\end{array}\right]\left[\begin{array}{c}
\bar{x}_{11}^{2}(k) \\
\bar{x}_{21}^{2}(k)
\end{array}\right]+\left[\begin{array}{c}
\widetilde{B}_{11}^{2} \\
\widetilde{B}_{21}^{2}
\end{array}\right] u(k),} \\
k \in Z_{+},
\end{gathered}
$$

where

$$
N_{1}=N_{2}=0 \text {, }
$$

$$
\begin{gathered}
{\left[\begin{array}{cc}
I_{n_{1}^{2}} & \widetilde{A}_{12}^{2} \\
\widetilde{A}_{21}^{2} & I_{n_{2}^{2}}
\end{array}\right]=\left[\begin{array}{ll}
1 & 2 \\
3 & 1
\end{array}\right],} \\
{\left[\begin{array}{c}
\widetilde{B}_{11}^{2} \\
\widetilde{B}_{21}^{2}
\end{array}\right]=\left[\begin{array}{c}
1 \\
0.182
\end{array}\right] .}
\end{gathered}
$$

In this case, from (29) for (30) we have

$$
\left[\begin{array}{l}
\bar{x}_{11}^{2}(k) \\
\bar{x}_{21}^{2}(k)
\end{array}\right]=\left[\begin{array}{c}
0.127 \\
-0.564
\end{array}\right] u(k), \quad k \in Z_{+} .
$$

Finally, the desired solution of the descriptor fractional linear system (6) with (23) is given by

$$
\left[\begin{array}{l}
x_{1}(k) \\
x_{2}(k)
\end{array}\right]=Q\left[\begin{array}{l}
\bar{x}_{1}^{1}(k) \\
\bar{x}_{1}^{2}(k) \\
\bar{x}_{2}^{1}(k) \\
\bar{x}_{2}^{2}(k)
\end{array}\right]=\left[\begin{array}{cc}
Q_{1} & 0 \\
0 & Q_{2}
\end{array}\right]\left[\begin{array}{l}
\bar{x}_{11}^{1}(k) \\
\bar{x}_{12}^{1}(k) \\
\bar{x}_{11}^{2}(k) \\
\bar{x}_{21}^{1}(k) \\
\bar{x}_{22}^{1}(k) \\
\bar{x}_{21}^{2}(k)
\end{array}\right],
$$

where

$$
\left[\begin{array}{l}
\bar{x}_{1}^{1}(k) \\
\bar{x}_{2}^{1}(k)
\end{array}\right] \text { and } \quad\left[\begin{array}{l}
\bar{x}_{1}^{2}(k) \\
\bar{x}_{2}^{2}(k)
\end{array}\right]
$$

are determined by (26) and (31), respectively.

\section{Concluding remarks}

The fractional discrete-time linear systems with two different fractional orders has been analyzed. The WeierstrassKronecker decomposition theorem of the regular pencil has been extended to the descriptor fractional discrete-time linear system with two different fractional orders. The method for finding the solution of the state equation has been derived. Effectiveness of the method has been demonstrated on a numerical example. Extension of these considerations on systems consisting of $n$ subsystems with different fractional orders is possible. An open problem is the application of the Drazin inverse to finding the solution of the system with different fractional orders.

Acknowledgements. This work was supported by the National Science Centre in Poland under the work No. 2014/13/B/ST7/03467.

\section{REFERENCES}

[1] S.L. Campbell, C.D. Meyer, and N.J. Rose, "Applications of the Drazin inverse to linear systems of differential equations 
with singular constant coefficients", SIAMJ Appl. Math. 31 (3), 411-425 (1976).

[2] L. Dai, Singular Control Systems, Lectures Notes in Control and Information Sciences, Springer-Verlag, Berlin, 1989.

[3] M. Dodig and M. Stosic, "Singular systems state feedbacks problems", Linear Algebra and Its Applications 431 (8), 1267 1292 (2009).

[4] D. Guang-Ren, Analysis and Design of Descriptor Linear Systems, Springer, New York, 2010.

[5] T. Kaczorek, "Descriptor fractional linear systems with regular pencils", Int. J. Appl. Math. Comput. Sci. 23 (2), 309-315 (2013).

[6] T. Kaczorek, "Singular fractional continuous-time and discretetime linear systems", Acta Mechanica et Automatica 7 (1), 2633 (2013).

[7] T. Kaczorek, "Singular fractional discrete-time linear systems", Control and Cybernetics 40 (3), 1-8 (2011).

[8] P. Van Dooren, "The computation of Kronecker's canonical form of a singular pencil", Linear Algebra and Its Applications 27, 103-140 (1979).

[9] P. Van Dooren and T. Beelen, "An improved algorithm for the computation of Kronecker's canonical form of a singular pencil”, Linear Algebra and Its Applications 105, 9-65 (1988).

[10] K. Nishimoto, Fractional Calculus, Decartess Press, Koriama, 1984

[11] K.B. Oldham and J. Spanier, The Fractional Calculus, Academmic Press, New York, 1974.

[12] I. Podlubny, Fractional Differential Equations, Academic Press, San Diego, 1999.

[13] A. Dzieliński, D. Sierociuk, and G. Sarwas, "Ultracapacitor parameters identification based on fractional order model", Proc. Eur. Control Conf. (ECC) 1, 196-200 (2009).

[14] N.M.F. Ferreira and J.A.T Machado, "Fractional-order hybrid control of robotic manipulators", Proc. 11th Int. Conf. Advanced Robotics, ICAR 1, 393-398 (2003).

[15] J.K. Popović, S. Pilipović, and T.M. Atanacković, "Two compartmental fractional derivative model with fractional derivatives of different order", Communications in Nonlinear Science and Numerical Simulation 18 (9), 2507-2514 (2013).

[16] K.S. Miller and B. Ross, An Introduction to the Fractional Calculus and Fractional Differenctial Equations, Willey, New York, 1993
[17] T. Kaczorek, "Solution of the state equations of descriptor fractional discrete-time linear systems with regular pencils", Tech. Transp. Szyn. 10, 415-422 (2013).

[18] T. Kaczorek, "Reduction and decomposition of singular fractional discrete-time linear systems", Acta Mechanica et Automatica 5 (4), 1-5 (2011).

[19] T. Kaczorek, "Application of Drazin inverse to analysis of descriptor fractional discrete-time linear systems with regular pencils", Int. J. Appl. Math. Comput. Sci. 23 (1), 29-33 (2013).

[20] T. Kaczorek, "Drazin inverse matrix method for fractional descriptor continuous-time linear systems", Bull. Pol. Ac.: Tech. 62 (3), 409-412, (2014).

[21] T. Kaczorek, "Fractional positive linear systems", Kybernetes: Int. J. Systems \& Cybernetics 38 (7/8), 1059-1078 (2009).

[22] T. Kazorek, Selected Problems in Fractional Systems Theory, Springer-Verlag, Berlin, 2011.

[23] T. Kaczorek, "Positive linear systems consisting of n subsystems with different fractional orders, IEEE Trans. Circuits and Systems 58 (6), 1203-1210 (2011).

[24] T. Kaczorek, "Positive linear systems with different fractional orders, Bull. Pol. Ac.: Tech. 58 (3), 453-458 (2010).

[25] M. Busłowicz, "Stability analysis of continuous-time linear systems consisting of n subsystem with different fractional orders", Bull. Pol. Ac.: Tech. 60 (2), 279-284 (2012).

[26] Ł. Sajewski, "Reachability, observability and minimum energy control of fractional positive continuous-time linear systems with two different fractional orders", Multidim. Syst. Sign. Process. 25, DOI 10.1007/s11045-014-0287-2 (2014).

[27] Ł. Sajewski, "Solution of the state equation of descriptor fractional continuous-time linear systems with two different fractional", Progress in Automation, Robotics and Measuring Techniques, Advances in Intelligent Systems and Computing 350, 233-242 (2015)

[28] Ł. Sajewski, "Descriptor fractional discrete-time linear system and its solution - comparison of three different methods", Proc. Conf. Automation 1, (2016), (to be published).

[29] T. Kaczorek, Vectors and Matrices in Automation and Electrotechnics, WNT, Warszawa, 1998.

[30] T. Kaczorek, Linear Control Systems, vol. 1, Research Studies Press J. Wiley, New York, 1992. 